\title{
Comparação da postura cervical de crianças e adolescentes com e sem disfunção temporomandibular
}

\author{
Comparison of the cervical position of children and adolescents with and without \\ temporomandibular dysfunction
}

\begin{abstract}
Alisson Sousa Santos ${ }^{1}$; Laryssa Castro Vale1; Paulo Henrique Martins de Sousa ; Guilherme Gonçalves Silva Pinto ${ }^{2}$; Ariane França Garçês ${ }^{3}$; Miguel Jânio Costa Ferreira ${ }^{4}$; Bruna Katarine Beserra $\mathrm{Paz}^{5}$; Ana Lourdes Avelar Nascimento ${ }^{6}$; Sarah Tarcisia Rebelo Ferreira de Carvalho7; Maria Claudia Gonçalves ${ }^{1,8}$
\end{abstract}

\begin{abstract}
Resumo: Avaliar as diferenças posturais entre crianças e adolescentes com e sem DTM. Foram inclusas 44 crianças e adolescente de ambos os sexos com idade de 10 a 15 anos, matriculados na Unidade de Ensino básico Luís Viana, São Luís - MA. Foram excluídos indivíduos que tinham realizado cirurgias ortognáticas prévias, que não apresentaram o termo de consentimento livre e esclarecido assinado pelo responsável e o termo de assentimento assinado ou que estivessem realizando tratamento para DTM. O diagnóstico da DTM foi feito pelo eixo I do RDC/TMD e a análise da postura corporal foi realizada pela fotogrametria computadorizada, com traçados dos ângulos de protrusão de cabeça (APC) e lordose cervical (ALC) e posteriormente analisados pelo software de análise fotográfica corporispro. Do total de 44 voluntários $55,12 \% \quad(n=13)$ apresentou diagnóstico de DTM miogênica, foi observada homogeneidade nos dados demográficas. Nos voluntários com DTM foi observada uma redução no APC em relação ao grupo sem DTM $(p<0,05)$, já para o ALC não foi observada diferença significativa entre os grupos $(P>0,05)$. Indivíduos com DTM apresentam maior retificação da coluna cervical e maior anteriorização de cabeça em relação a indivíduos sem DTM. Esses achados chamam a atenção para a importância da avaliação da postura craniocervical em crianças com diagnóstico ou mesmo com sinais e sintomas de DTM.
\end{abstract}

Palavras-chave: Postura cervical; Disfunção temporomandibular; fotogrametria.

\begin{abstract}
To evaluate postural differences among children and adolescents with and without TMD. We included 44 children and adolescents of both sexes, aged 10 to 15 years, enrolled in the Luís Viana Elementary School, São Luís - MA. We excluded individuals who had previous orthognathic surgeries, who did not present the informed consent form signed by the person in charge and the agreement signed or who were performing treatment for TMD. The diagnosis of TMD was made by the axis of the Diagnostic Criteria in Research for Temporomandibular Dysfunction (RDC / TMD) and the analysis of body posture was performed by computerized photogrammetry, with labeling of the anatomical points corresponding to the head protrusion angles (ALC) and lordosis Cervical (ALC) and later analyzed by the corporispro photographic analysis software. A total of 44 volunteers $(n=13)$ presented a diagnosis of myogenic TMD, there were homogeneity of the mean values and standard deviations of the demographic variables, in the volunteers with TMD a reduction was observed in the APC compared to the group without TMD $(P<0.05)$. However, for the LAC, no significant difference was observed between the groups $(P>0.05)$, although an angular increase of the TMD group was observed in relation to the control group. Individuals with TMD present greater cervical rectification and greater anteriority of the head in relation to individuals without TMD. These findings point to the importance of assessing craniocervical posture in children diagnosed or even with signs and symptoms of TMD.
\end{abstract}

\footnotetext{
${ }^{1}$ Discente em fisioterapia pela Universidade CEUMA.

2 Discente do Curso de Medicina das Faculdades Integradas da União Educacional do Planalto CentralFACIPLAC.

${ }^{3}$ Discente do Programa de Pós-graduação em Meio Ambiente da Universidade CEUMA.

${ }^{4}$ Docente do curso de graduação em Fisioterapia da Universidade CEUMA

${ }^{5}$ Doutora em Medicina pela Universidade de São Paulo; Docente do mestrado em Meio Ambiente e do curso de graduação em Fisioterapia da Universidade CEUMA
}

Autor responsável: Maria Claudia Gonçalves: Rua da Sociologia, noo6, COHAFUMA, São Luís-MA

Rev. Investig, Bioméd. São Luís, 9(2): 138-148, 2017 
Keywords: Cervical posture; Temporomandibular dysfunction; Photogrammetry. Introdução

A postura é definida pela relação homeostática das estruturas musculoesqueléticas e demais estruturas correlacionadas, que se adaptam ao recebimento de estímulos ${ }^{1}$ tendo como característica a realização de atividades com pouco gasto energético ${ }^{2}$. Portanto, os desarranjos das estruturas musculoesqueléticas podem ocasionar alterações posturais ${ }^{3}$.

Hiperlordose cervical e lombar, hipercifose dorsal, retificação cervical, dorsal e lombar são descrita como alterações posturais decorrentes de anormalidades nas curvaturas da coluna vertebral, podendo ser acompanhada de uma protrusão de cabeça, o que pode repercutir com alteração da articulação temporomandibular (ATM) ${ }^{4}$.

A Disfunção Temporomandibular (DTM) é um termo genérico caracterizado por um conjunto de alterações que envolvem desde a ATM, estruturas associadas e os músculos mastigatório ${ }^{5}$. Sua manifestação é comum em adultos ${ }^{6}$, porém, devido as grandes modificações osteomioarticulares durante o crescimento craniofacial, crianças e adolescente podem manifestar sinais e sintomas de DTM ${ }^{7}$.

Além das modificações osteomioarticulares, a manifestação da DTM, em especial na infância pode estar associada a frequência de hábitos parafuncionais orais e viciosos que afetam a oclusão ${ }^{8}$ músculos da mastigação, ombro e cervical ${ }^{9,10}$. Mediante a isto, tais alterações funcionais nessas estruturas podem ocasionar alterações na postura e a instabilidade da cervical ${ }^{11}$.

$\mathrm{Na}$ literatura são descritos alguns recursos para avalição da postura de forma qualitativa e quantitativa. Como recursos qualitativos pode-se citar: a fotografia onde foi analisada visualmente e classificada as alterações da curvatura lordose cervical e lombar $^{12}$. O acromiopelviômetro recurso que marca a posição da pelve e ombro para verificar a presença de assimetrias ${ }^{13}$, entre outros.

Já como recursos quantitativos podese destacar a radiografia, que é mais reprodutível quando comparado a fotografia e vídeo. No entanto, apesar do raio-x ser um método eficaz para análise das alterações da curvatura da coluna vertebral, sua utilização requer gastos para sua realização, além de se tratar de um recurso radioativo não devendo ser realizado com frequência além de apresentar contra-indicações como por exemplo em gestantes ${ }^{14}$.

A fotogrametria é uma palavra de origem grega e significa medir graficamente usando a luz, sendo descrita também como uma forma de quantificar as alterações posturais. Possui algumas divisões quanto ao tipo, dentre estas a fotogrametria médica também conhecida como bioestereometria que mensura as formas, dimensões dos corpos e/ou parte deste. É um método eficaz, não invasivo que não gera grandes custos para sua realização além de não apresentar contraindicações, na fotogrametria a fotografia é tirada e depois analisada em um software específico ${ }^{15}$.

Atualmente, a fotogrametria vem sendo utilizada em várias áreas da saúde como na odontologia ${ }^{16}$, na cirurgia plástica $^{17}$ e na fisioterapia ${ }^{18}$.

$\mathrm{Na}$ fisioterapia, a fotogrametria é comumente utilizada para análise postural, como por exemplo nos estudos que avaliaram o ângulo $Q$ em diferentes posturas estáticas de homens $e$ mulheres $^{19}$, e a correlação entre a dor anterior do joelho e a medida do ângulo $Q$ pela fotogrametria computadorizada ${ }^{20}$. Ambos os estudos afirmaram a fotogrametria como um método eficaz de 
medição angular em relação a outros métodos citados pela literatura ${ }^{21}$.

$\mathrm{Na}$ literatura a fotogrametria já fora utilizada para análise postural de crianças com DTM, onde comparou-se dois ângulos cervicais com a oclusão em crianças com e sem DTM, resultando em associação com a condição oclusal em um dos ângulos avaliados e não houve associação dos dois ângulos avaliados com a DTM ${ }^{22}$, todavia, os ângulos avaliados foram diferentes do presente estudo.

Dessa forma, o presente estudo apresentou como problema a seguinte indagação: Quais as diferenças da postura cervical em crianças e adolescente com e sem disfunção temporomandibular? Tendo como objetivo geral: avaliar as diferenças posturais de crianças e adolescentes com e sem DTM.

\section{Materiais e métodos}

O presente estudo foi de cunho transversal. Pois observou a presença ou ausência de exposição ao fator e à consequente presença ou ausência da doença, tendo hipótese, como característica de estudos analíticos ${ }^{23}$. Participaram deste estudo 44 crianças e adolescente de ambos os sexos, com idade de 10 a 15 anos, foram excluídos indivíduos que tivessem realizado cirurgias ortognáticas anterior ao dia da avaliação, que realizaram ou estivessem realizando tratamentos ortodônticos ou para DTMs, que não apresentaram 0 termo de consentimento livre e esclarecido (TCLE) assinado pelos pais e termo de assentimento assinado pelo aluno, e que não estivessem regularmente matriculados na escola Luís Viana, local onde foi realizada a coleta de dados, situada na cidade de São Luís - MA.

$\mathrm{Na}$ avaliação foi utilizado os seguintes materiais:
- Máquina fotográfica Canon Rebeu I5

- Etiquetas de papel

- TNT escuro

- Tripé

- Paquímetro

- Algodão

- Luvas de procedimento

- Álcool 70\%

- Questionários próprios de avaliação

As luvas de procedimentos e o paquímetro eram utilizados para avaliar a amplitude de movimento mandibular bem como para a inspeção da cavidade oral. As etiquetas foram utilizadas para marcar os pontos anatômicos necessários para traçar os ângulos de interesse.

A priori foi esclarecido os objetivos do estudo para diretoria e para os professores da escola, após o devido esclarecimento foi enviado aos pais e responsáveis um TCLE e o termo de assentimento para ser assinado pelas crianças, onde constou todos os propósitos, objetivos e procedimentos da pesquisa, que deveria ser assinado e entregue no dia da avaliação caso permitida a participação do aluno. Inicialmente foi realizada uma coleta dos dados gerais como idade, sexo, cor da pele referida e série que cada criança pertencia e antropométricos, peso, altura, afim de caracterizar a amostra estudada.

Para que o avaliador fosse blindado em relação ao diagnóstico de DTM a fotogrametria, registro e análise das fotos, foi realizada sem que o avaliador tivesse 0 conhecimento do diagnóstico de DTM, para tanto a avaliação da DTM foi feita somente depois do registro fotográfico e a análise das fotos foi realizada por um segundo avaliador que não tinha conhecimento do diagnóstico de DTM.

Os voluntários foram avaliados, em local reservado, por meio de registros fotográficos em posição ortostática nos perfis anterior, posterior e laterais (Imagens A, B, C e D). 
A

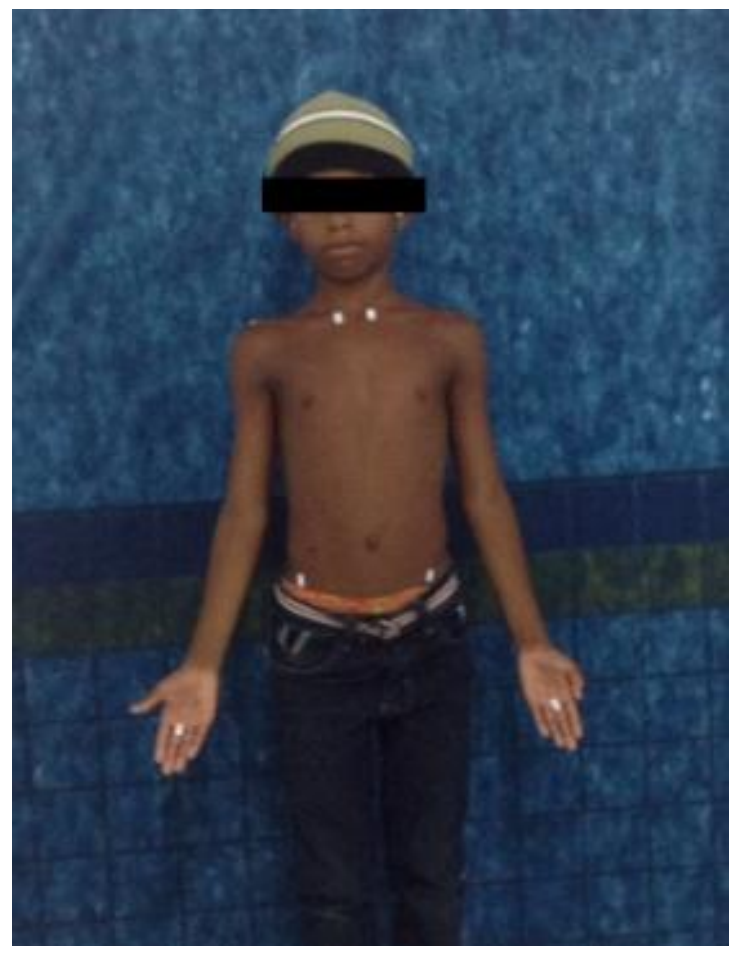

C

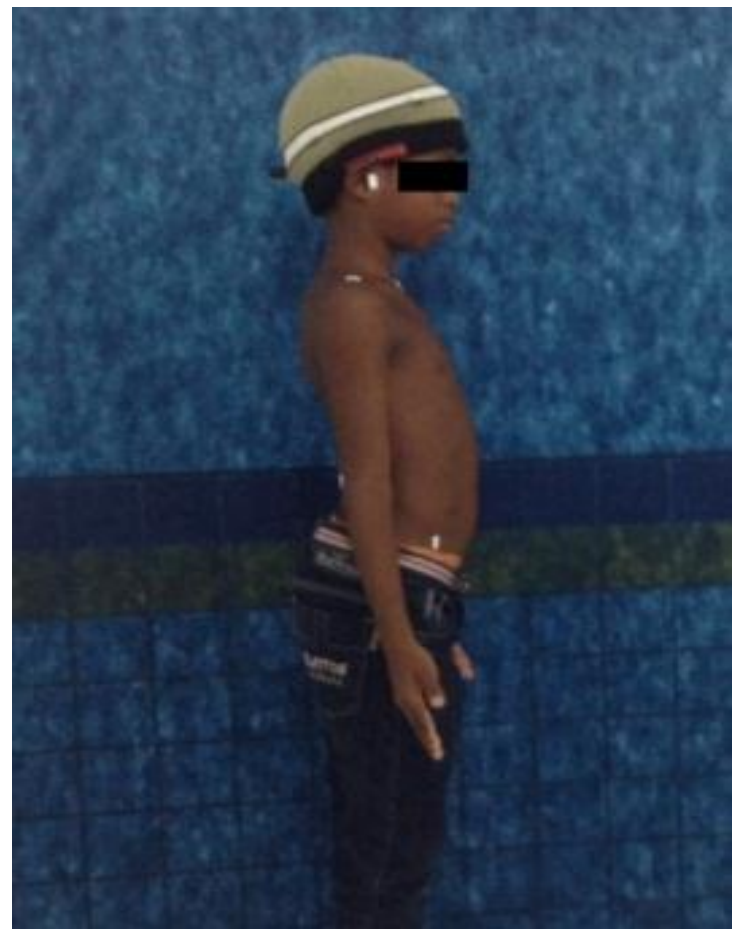

B

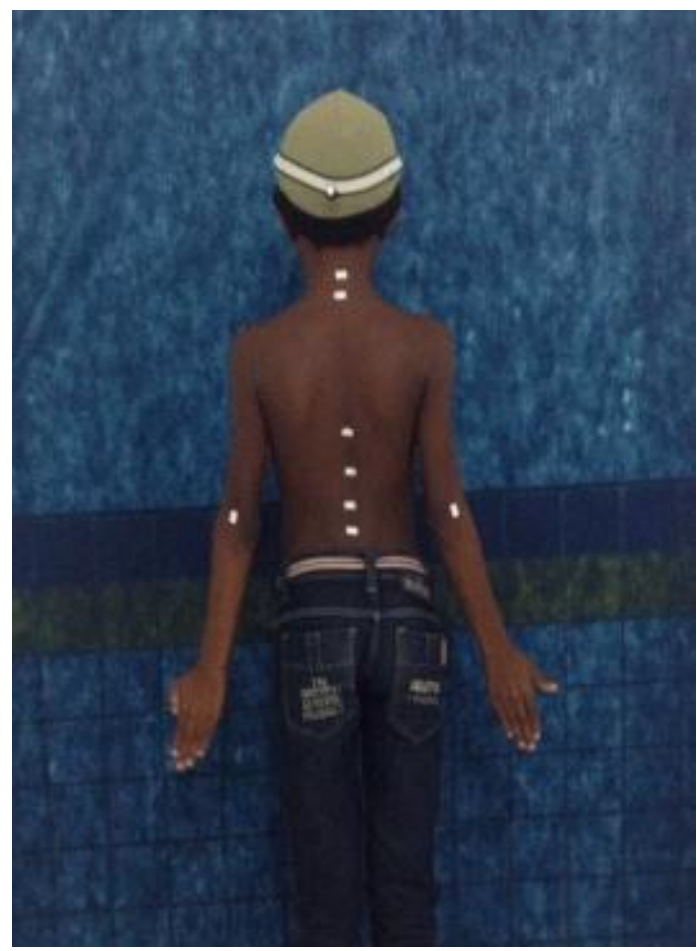

D

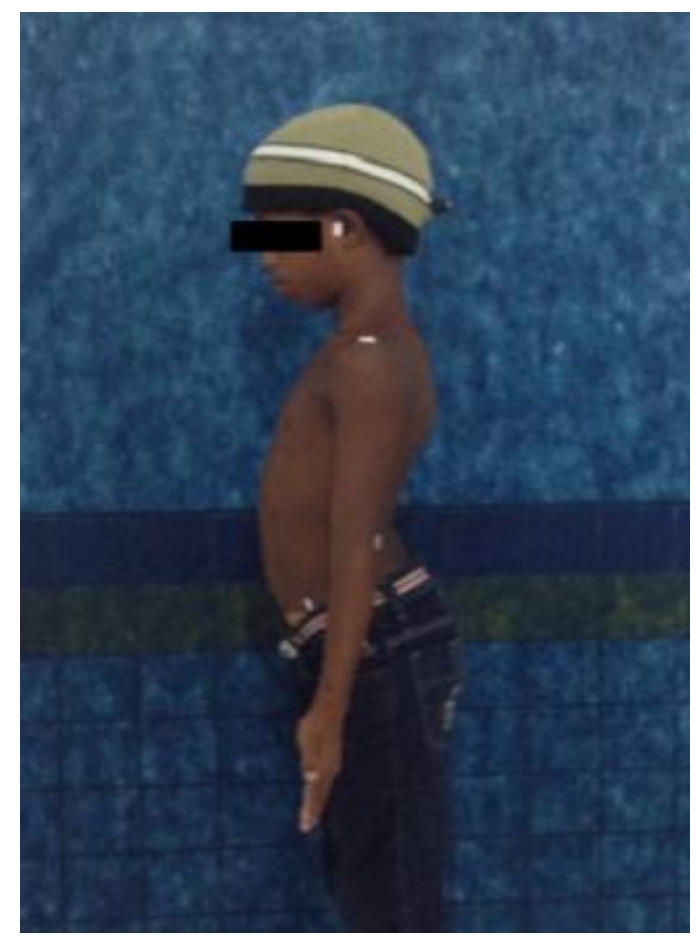

Imagens: $\mathrm{A}=$ Frontal, $\mathrm{B}=$ Posterior, $\mathrm{C}=$ Lateral direita, $\mathrm{D}=$ Lateral esquerda 
Os voluntários foram orientados a direcionar o olhar para um ponto fixo na parede a uma distância de 1 metro do voluntário e, após breve caminhada pela sala de exame, os voluntários foram posicionados no local de registro fotográfico. A máquina fotográfica foi posicionada sobre um tripé fotográfico, cujo centro foi posicionado a uma distância fixa de 2,40 metros do voluntário ${ }^{24}$, o centro da lente da máquina fotográfica foi posicionado no centro do abdômen do indivíduo, sendo utilizada uma objetiva de $105 \mathrm{~mm}^{25}$. O método para obtenção e registro das imagens foi conforme um manuscrito da literatura ${ }^{26}$.

Foram traçados os ângulos APC (protrusão de cabeça) e ALC (lordose cervical) (Imagens E e F). Pontos anatômicos considerados: 1- Articulações Esternoclaviculares, 2- Espinhas llíacas Antero Superiores, 3- Tragus da orelha, 4Acrômio da escápula, 5- Epicôndilo Lateral do cotovelo, 6- Art. metacarpofalangeana do $3^{\circ}$ dedo, 7- Protuberância Occipital, 83 ${ }^{\text {a }}$ Vértebra Cervical, 9- $7^{\text {a }}$ Vértebra Cervical, 10- $7^{\mathrm{a}}$ Vértebra Torácica, 11- 12 ${ }^{\mathrm{a}}$ Vértebra Torácica, 12- 3a Vértebra Lombar, 13- $5^{\text {a }}$ Vértebra Lombar ${ }^{27}$.

O diagnóstico de DTM foi realizado utilizando o eixo I do Critério diagnóstico em pesquisa RDC/TMD, sendo aplicado por um único examinador treinado, como recomendado pelo consórcio internacional ${ }^{28}$. A ferramenta consistiu em um roteiro de avaliação clínica ostensivamente utilizada na pesquisa ${ }^{28}$. $O$ RDC/TMD consistiu na mensuração da amplitude de movimento mandibular, avaliação da dor à palpação, além da observação de estalidos e crepitações ao movimento ${ }^{29}$.
As variáveis quantitativas foram descritas por média e desvio padrão (média \pm DP) e as qualitativas foram apresentadas em frequência. Os dados foram analisados no software Statistical Package for the Social Sciences (SPSS 18.0). A diferença das médias dos ângulos entre os grupos foi comparada usando a análise de variância (ANOVA). O nível de significância estatística de $p \leq 0,05$ foi adotado.

Este projeto foi aprovado pelo Comitê de Ética em pesquisa da Universidade CEUMA, todos os pais e responsáveis assinaram o termo de Consentimento Livre e esclarecido e todas as crianças assinaram o termo de assentimento, de acordo com as diretrizes e normas reguladoras da pesquisa envolvendo seres humanos do Conselho Nacional de Saúde, Resolução 466/2012. Aos participantes do estudo foram garantido 0 direito de interrupção do estudo a qualquer momento, o acesso aos resultados, o sigilo sobre os mesmos, orientações e encaminhamentos para avaliação especializada quando se fizesse necessário.

\section{Resultados}

Foram avaliados 44 alunos do $6^{\circ}$ ao 9o ano da escola Luís Viana situada na cidade de São Luís - MA. Dos 44 alunos avaliados $55,12 \% \quad(n=13)$ apresentaram diagnostico de DTM miogênica segundo o RDC/TMD. Cerca de 59,09\% ( $n=26)$ pertenciam ao gênero feminino. Não foram observadas diferenças significativas entre os grupos avaliados quanto as variáveis idade, peso e altura (Tabela 1). 


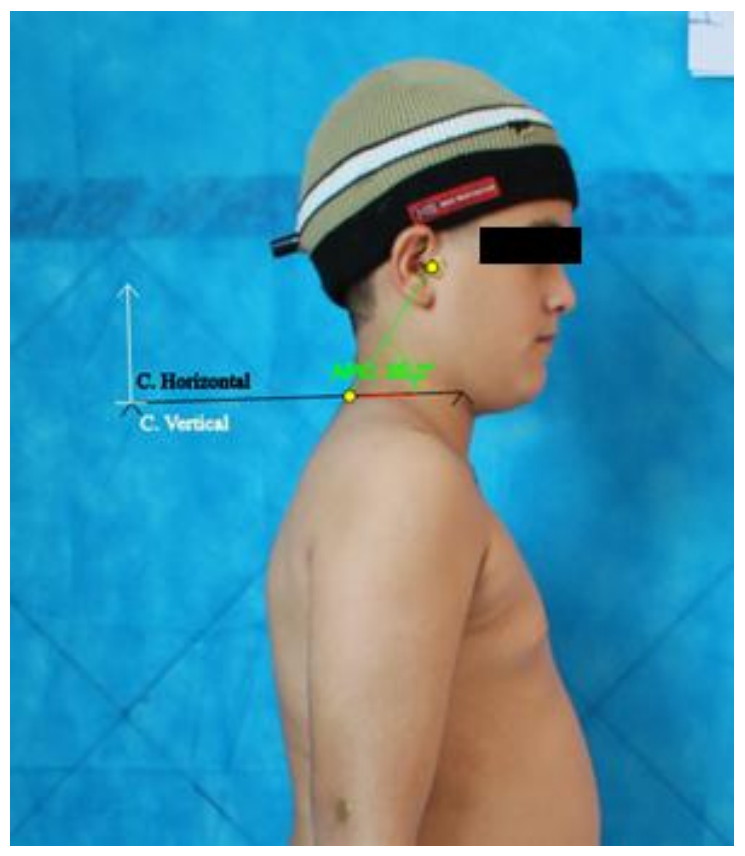

Imagem E: APC - processo espinhoso de $\mathrm{c7}$ e tragus da orelha

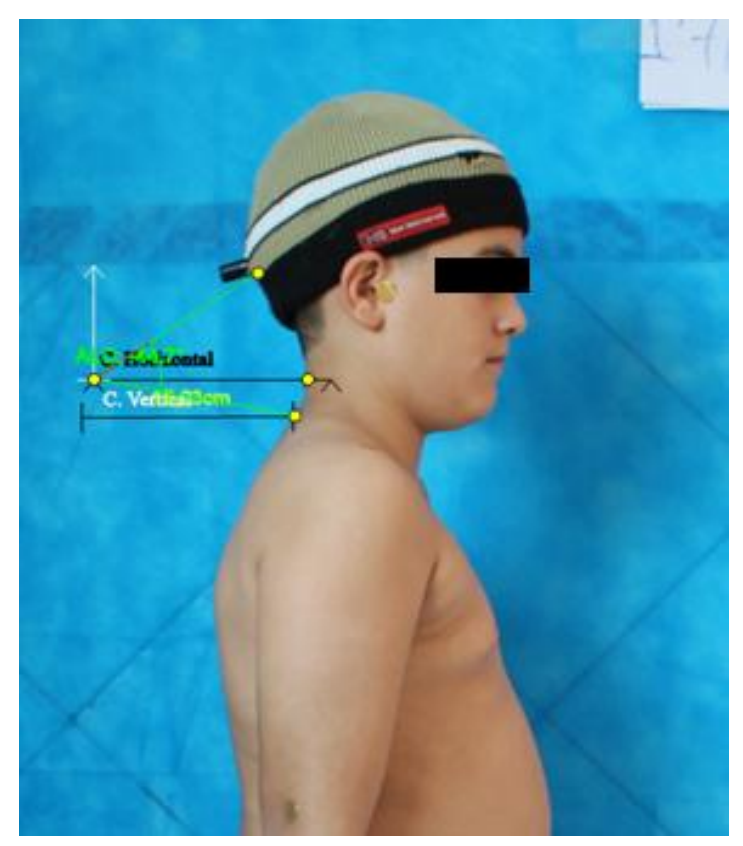

Imagem F: ALC - processo espinhoso de c7, c3 e protuberância occcipital 
Tabela 1. Valores médios e desvios padrões dos dados demográficos e frequência em porcentagem do número de indivíduos com diagnóstico de DTM segundo o RDC distribuído por gênero $(n=44)$.

\begin{tabular}{cccccc}
\hline Gênero & $\begin{array}{c}\text { Idade } \\
(\text { anos })\end{array}$ & $\begin{array}{c}\text { Peso } \\
(\mathrm{kg})\end{array}$ & $\begin{array}{c}\text { Altura } \\
\left(\mathrm{cm}^{2}\right)\end{array}$ & $\begin{array}{c}\text { DTM } \\
(\%)\end{array}$ & p-valor \\
\hline Feminino $(\mathrm{n}=26)$ & $13,07(1,46)$ & $53(5)$ & $1,55(0,20)$ & $38,46 \%(10)$ & $\mathrm{p}>0,05$ \\
\hline Masculino $(\mathrm{n}=18)$ & $12,76(1,5)$ & $51(2)$ & $1,50(0,30)$ & $16,66 \%(3)$ & \\
\hline
\end{tabular}

Embora tenham sido marcados diversos pontos, neste trabalho são demonstrados e discutidos somente os ângulos craniocervicais de APC e ALC.

O principal objetivo deste trabalho foi avaliar se existia diferenças posturais da região craniocervical entre crianças e adolescentes com e sem DTM, nossa hipótese foi comprovada. Nos voluntários com DTM foi observada uma redução no APC em relação ao grupo sem DTM sendo atribuída uma diferença significativa $(p<0,05)$. Em contrapartida para - ALC, embora tenha sido observada um aumento angular do grupo DTM em relação ao controle, não foi observada diferença significativa entre os grupos $(P>0,05)$, como pode ser observado na Tabela 2.

Tabela 2. Valores médios e intervalo de confiança (95\%) dos 2 ângulos (graus) avaliados com e sem DTM $(n=44)$.

\begin{tabular}{|c|c|c|c|}
\hline & Com DTM $(n=13)$ & Sem DTM $(n=31)$ & \\
\hline Ângulos & Média (IC) & Média (IC) & $\mathrm{P}$ (valor) \\
\hline${ }^{*} \mathrm{APC}$ & $49.1(44.20-50.15)$ & $53.2(51.15-55.30)$ & $p<0,05$ \\
\hline${ }^{*} \mathrm{ALC}$ & $53,5(49.2-55.40)$ & $51(48.70-56.30)$ & $p>0,05$ \\
\hline
\end{tabular}

\section{Discussão}

A maior porcentagem de diagnóstico de DTM encontrada no gênero feminino neste estudo corrobora com a literatura. Onde foi observado maior prevalência de DTM $28,1 \%$ em adolescentes do gênero feminino ${ }^{30}$, e maior prevalência, $57 \%$, de sinais e sintomas de DTM e hábitos parafuncionais em estudantes universitários do gênero feminino ${ }^{31}$, mesmo tendo sido usado o índice anamnésico de Fonseca para determinar a prevalência.

$\mathrm{Na}$ literatura sugerem-se que essa maior prevalência no gênero feminino seja devido ao fato das mulheres serem mais atenciosas e cuidadosas com a saúde e por isso procuram por atendimento médico com mais frequência que os homens ${ }^{32}$. Ou que as diferenças anatomo-fisiológicas do sexo feminino como diferenças hormonais, 
das estruturas musculares e limiar de dor menor, são fatores que determinam essa maior prevalência de DTM em mulheres $^{33,34}$, contudo ressaltam a necessidade de maiores investigações sobre o assunto.

Existe ampla evidência na literatura sobre a relação entre postura craniocervical e DTM, principalmente em adultos. Alguns são positivos ${ }^{35,36}$ e alguns são negativos ${ }^{37,38}$ e alguns ainda sugerem que o posicionamento anterior pode ser uma consequência da DTM em vez de sua causa $^{39}$. A população estudada e os métodos empregados podem contribuir para esses resultados variados.

Entretanto, em se tratando da postura craniocervical de crianças e adolescentes com e sem DTM, utilizando a fotogrametria, existe escassez de trabalhos que tenham avaliados o que limita a discussão dos dados deste estudo com outros que tenham amostras e métodos semelhantes.

Motta et al. (2011) Comparando crianças de 3 a 6 anos com bruxismo, avaliadas por meio da fotogrametria, observaram maiores alterações na postura da cabeça das crianças com bruxismo em relação ao controle ${ }^{40^{3}}$.

Ainda, em crianças e adolescentes de 8 e 14 anos foi observado melhor perfil postural global de crianças asmáticas em relação a crianças não asmáticas, no entanto, para este estudo não foi avaliado ângulos cervicais na vista lateral ${ }^{41}$ e em crianças respiradoras bucais foi observado uma diminuição no ângulo de protrusão de cabeça, o que repercuti em anteriorização da mesma ${ }^{42}$.

Não foi observada diferenças significantes para os ângulos APC e ALC em um estudo que avaliaram crianças com e sem $D_{T M}^{22}$. Essa diferença em relação aos resultados apontados neste estudo pode ser atribuída a diferenças metodológicas, principalmente o método utilizado para avaliar a postura e os pontos anatômicos utilizados para obter os ângulos de interesse.

Em contrapartida, nossos dados corroboram por um estudo que avaliou crianças e adolescentes por meio da cefalometria radiográfica e encontrou correlação positiva entre sinais e sintomas de DTM e anteriorização de cabeça ${ }^{39}$ e com um estudo quando comparado a adultos com DTM que observaram uma redução significativa no APC de mulheres adultas com $\mathrm{DTM}^{27}$. A redução do APC indica maior retificação da coluna cervical e maior anteriorização da cabeça o que pode alterar $o$ centro de gravidade $e$ promover alterações posturais em todo o corpo.

Foi identificada relevância estatística corroborando com nosso estudo em um manuscrito ao analisar a relevância clínica para os parâmetros de postura craniocervical em adultos com DTM comparados ao grupo controle, porém não foi identificada relevância clínica ${ }^{43}$. Dessa forma, a redução significativa do APC e o aumento angular do ALC observadas no grupo com DTM devem ser analisados com atenção quando usados para avaliar a melhora clínica dos pacientes uma vez que somente a diferença estatística foi comprovada.

Uma limitação deste estudo foi à dificuldade de se obter consentimento das escolas e dos pais para realizar as avaliações e a timidez dos voluntários que desistiam de participar por terem que ficar parcialmente despidos no momento da realização da fotografia, resultando em um tamanho amostral reduzido. $O$ desenho transversal não permite estabelecer relação de causa e efeito entre a DTM e a alteração postural encontrada.

Entretanto, a adoção de ferramentas acuradas e não invasivas, como o RDC/TMD e a fotogrametria, para avaliação da DTM e da postura, o rigor 
metodológico de um avaliador blindado, previamente treinado para a marcação dos pontos anatômicos e tomada e análises fotográficas reforçam a relevância e originalidade deste manuscrito. Ainda, o conhecimento desses fatores é importante para guiar a decisão terapêutica de fisioterapeutas e dentistas.

\section{Conclusão}

Os resultados do presente estudo demonstraram a diferença na postura craniocervical entre crianças e adolescentes com DTM e um grupo controle. Indivíduos com DTM apresentam maior retificação da coluna cervical e maior anteriorização de cabeça em relação a indivíduos sem DTM. Esses achados chamam a atenção para a importância da avaliação da postura craniocervical em crianças com diagnóstico ou mesmo com sinais e sintomas de DTM. Estudos longitudinais são necessários para estabelecer uma relação causal entre alteração postural e DTM e confirmar os presentes achados.

\section{Agradecimentos}

Este trabalho foi contemplado com auxilio edital Universal processo 00208/15 e bolsa de Iniciação Científica pela Fundação de Amparo ao desenvolvimento Científico e Tecnológico do estado do Maranhão (FAPEMA).

\section{Referências}

1 Contri, D.E.; Petrucelli A; Perea D.C. Incidência de desvios posturais em escolares de $2^{\circ}$ ao $5^{\circ}$ ano do Ensino Fundamental. ConScientiae Saúde, v. 8, n. 2, p. 219-224, 2009.

2 Tanak, C.; Farah, E.A. Anatomia Funcional das Cadeias Musculares. São Paulo: Ícone editora, 1997.

3 Knoplich, J. Enfermidades da Coluna Vertebral. 3. Ed. São Paulo: Panamed, 2002.

4 Kisner, C; Colby, L.A. Exercícios Terapeuticos. São Paulo: Editora Manole,2015.

5 De Oliveira, A.S. et al. Prevalence study of signs and symptoms of temporomandibular disorder in Brazilian college students. Braz Oral Res, São Paulo, v. 20, n. 1, 2006.

6 Gonçalves, D.A. et al. Temporomandibular symptoms, migraine and chronic daily headaches in the population. Neurology, $v$. 25, n. 73, p. 645-646, 2009.

7 Alamoudi, N. et al. Temporomandibular disorders among school children. J Clin Pediatr Dent. Summer; v. 22, n. 4, p. 323-8, 1998.

8 Guimarães, M.M.B.; Sacco, I.C.N.; João, S.M.A. Caracterização postural da jovem praticante de ginástica olímpica. Rev. Brás. Fisioterap, São Carlos, v. 11, n. 3, p. 213219, 2007.

9 Abraao, S.D.; Fornasari, C.A. Avaliação da posição da cabeça e plano de Frankfut na disfunção da ATM por meio de fotometria. RBO, v. 62, n. 1e2, p. 82-4, 2005.

10 Vélez, A.L. et al. Head posture and dental wear evaluation of bruxist children with primary teeth. Journal of Oral Rehabilitation; v. 34, p. 663-70, 2007.

11 Faraco, C.C.; Mattos H.M. Desvios posturais em indivíduos com maloclusoes Classe I, Classe II e Classe III. Rev. Ter. Man; v. 23, p. 28-31, 2008. 
12 Fedorak, C. et al. Reability of the visual assessment of cervical and lumbar lordoses: how good are we? Spine, Philadelphia, v. 28, n. 16, p. 1857-59, 2003.

13 Fuentes, R.; Freesmeyer, W.; Henríquez J. Influencia de la postura corporal em la prevalência de lãs disfunciones craneomandibulares. REV. MÉD. Chile, v. 127, n. 9, p. 1079-1085, 1999.

14 Benson, P.E.; Richmond A.S. A critical appraisal of mensurement of the solf tissue outline using photographs and vídeo. Eur. J. Orthod., Oxford; v,19, n 4, p. 397-409, 1997.

15 Tommaselli, A.M. et al. Fotogrametria: Aplicações a curta distância. FCT 40 anos Perfil Cientifico Educacional. Presidente: Meneguetti Jr e Alves, 1999.

16 Ferrario, V.F. et al. New televisión techinique for natural head and body posture análisis. The J. of Craniomand. Practice, v.13, n. 4, p. 247-55, 1995.

17 Hochman, B; Castilho, H.T; Ferreira, L.M. Padronização fotográfica e morfométrica na fotogrametria computadorizada do nariz. Acta Cir. Bras., São Paulo; v.12, n.4, p. 25866, 2002.

18 Baraúna, M.A. et al. Estudos do equilíbrio estático de idosos e sua correlação com quedas. Fisioterapia Brasil, v. 5, n. 2, p. 136-141, 2004.

19 Sanchez, H.M. Avaliação do ângulo Q em diferentes posturas estáticas. Acta Ortop Bras, v. 22, n. 6, p. 325- 329, 2011.

20 Melo, P.G. et al. Correlação entre a dor anterior do joelho e a medida do ângulo "q" por intermédio da fotometria computadorizada. Rev. bras. Fisioter; v. 8, n.1, p. 39-43, 2004.

21 Souza, J.A. et al. Global body posture and plantar pressure distribution in individuals with and without temporomandibular disorder: a preliminary study. J Manipulative Physiol Ther; v. 37, n. 6, p. 407-14, 2014.

22 Biasotto-Gonzalez, D.A. et al. Analise comparativa entre dois ângulos cervicais com a oclusão em crianças com e sem
DTM. Rev. CEFAC, São Paulo, v.14, n.6, p.1146-1152, 2012.

23 Haddad, N. Metodologia de estudos em ciências da saúde. Sao Paulo: Roca; 2004.

24 Nayler, J.R. Clinical Photography: A guide for the clinician. Postgrad Med; v. 49, p. 256-62, 2003.

25 Claman, L.; Patton, D.; Rashid, R. Standardized portrait photography for dental patients. Am J Orthod. Dentofac Orthop, v. 98, p. 197-205, 1990.

26 Belli, J.F.C. et al. Analysis of body posture in children with mild to moderate asthma. Eur J Pediatr, v.168, p.1207-1215, 2009.

27 Ferreira, M.C. et al. Body posture changes in women with migraine with or without temporomandibular disorders. Braz J Phys Ther, v. 18, p.19-29, 2014.

28 Dworkin, S.F.; Leresche L. Research diagnostic criteria for temporomandibular disorders: review, criteria, examinations and specifications, critique. J Craniomandib Disord.1992 Fall;6(4):301-55

29 Chaves, T.C.; Oliveira, A.S.; Grossi, D. B. Principais instrumentos para avaliação da disfunção temporomandibular, parte I: índices e questionários; uma contribuição para a prática clínica e de pesquisa. Fisioter. Pesqui. vol.15 no.1 São Paulo 2008

30 Fernandes, G. et al. Parafunctional habits are associated cumulatively to painful temporomandibular disorders in adolescentes. Braz. oral res.vol.30 no.1 São Paulo 2016.

31 Medeiros, S.P. et al. Prevalência de sintomas de disfunção temporomandibular e hábitos parafuncionais em estudantes universitários. Rev Gaúcha Odontol. Porto Alegre, v.59, n.2, p.201-208, abr./jun., 201.

32 Pereira, K.N. et al. Sinais e sintomasde pacientes com disfunção temporomandibular. Rev CEFAC. 7(2):2218, 2005.

33 Feteih RM. Signs and symptoms of temporomandibular disorders and oral parafunctions in urban Saudi Arabian 
adolescents: a research report. Head Face Med. 25(2):1-7, 2006.

34 Pedroni, C.R.; Oliveira, A.S.; Guaratini, M.I. Prevalence study of signs and symptoms of temporomandibular disorders in university students. J Oral Rehabil. 30(2):283-9, 2003.

36 Lee, W.Y.; Okeson, J.P.; Lindroth, J. The relationship between forward head posture and temporomandibular disorders. J Orofac Pain. 9:161-67, 1995.

35 Armijo-Olivo, S. et al. The association between 2the cervical spine, the stomatognathic, system and craniofacial pain: a critical review. J Orofac Pain. 20(4):271-87, 2006.

37 Visscher, C.M. et al. Is there a relationship between head posture and craniomandibular pain? J Oral Rehabil. 29(11):1030-6,2001.

38 lunes, D.H. et al. Craniocervical posture analysis in patients with temporomandibular disorder. Rev Bras Fisioter.13(1):89-95, 2009.

39 Sonnesen, L.; Bakke, M.; Solow, B. Temporomandibular disorders in relation to craniofacial dimensions, head posture and bite force in children selected for orthodontic treatment. Eur J Orthod. Apr;23(2):179-92, 2001.

40 Motta, L.J. et al. Craniocervical Posture and Bruxism in Children. Physiother. Res. Int. Mar;16(1):57-61. doi: 10.1002/pri.478, 2011.

41 Ricieri, D.V.; Costa, J.R.; Filho, N,A,R. Impacto da asma sobre a postura corporal de crianças entre 8 e 14 anos analisada pela biofotogrametria. ACTA FISIATR, 15(4): $214-219,2008$.

42 Weber, P. et al. Análise da postura craniocervical de crianças respiradoras bucais após tratamento postural em bola suíça. Fisioter Pesq. 19(2):109-14, 2012.
43 Armijo-Olivo, S. et al. Clinical relevance vs. statistical significance: Using neck outcomes in patients with temporomandibular disorders as an example. Man Ther.16(6):563-72, 2011. 